\title{
Dinâmica de um Sistema Predador-Presa com Ocorrência de Doença Contagiosa na População de Presas.
}

\author{
Danilo F. F. Leonel, Carlos Henrique dos Santos, \\ Liliana M. Gramani, Luiz Antonio R. de Santana \\ PPGMNE, Depto. de Matemática, Depto. de Matemática, Depto. de Matemática - UFPR, \\ 81531-980, Curitiba, PR \\ E-mail:daniloleonel@ufpr.br, cahe@ufpr.br, lgramani@ufpr.br, lars@ufpr.br
}

\begin{abstract}
Resumo: Neste trabalho, estudamos um modelo matemático contínuo de situação ecoepidemiológica de predação seletiva. Após adimensionalizar as equações correspondentes, consideramos a análise sob o ponto de vista de estabilidade linear, do modelo que trata da interação das populações de presas suscetiveis $(S)$, presas infectadas $(I)$ e predadores $(P)$, onde a infecção ocorre por contato diretamente proporcional com a população infectada. Optamos por alterar num dos sistemas, proposto em [3], a modelagem feita para o crescimento de predadores, tendo em vista que o comportamento populacional mais interessante para se estudar é o da população de presas, por causa da predação ocorrer na população que é suscetível à doença.
\end{abstract}

Palavras-chave: Predação Seletiva, Predador-Presa, Ecoepidemiologia.

\section{Formulação do modelo matemático}

A modelagem e a análise de um sistema predador-presa com a situação de predação seletiva na ocorrência de doença contagiosa é o objetivo deste trabalho. A predação ocorre pela preferência aos indivíduos não infectados por uma determinada doença. Seguimos as suposições feitas pelo artigo [3], mas propomos uma modeladem da população de predadores diferente, como veremos a seguir.

Para representar essa situação, foi levada em consideração que a população de presas está dividida em duas classes quando na ocorrência da doença. Seriam $(S)$ os indivíduos suscetíveis à doença, ou seja, a parcela saudável da população total; e $(I)$ os indivíduos infectados pela doença. Supondo que a população contaminada pela doença não seja capaz de se reproduzir, então a variação da densidade populacional de presas ocorrerá apenas dentre os indivíduos suscetíveis. Também se releva a capacidade ambiental $K$ para um determinado número de indíviduos e portanto o crescimento da população suscetível é admitido como sendo logístico. A taxa de crescimento intrínseco da população de presas suscetíveis é descrita pela constante $r$. É importante observar que a limitação para o crescimento leva em consideração a população total de presas, porque se supõe que os indivíduos infectados também competem por alimento e espaço com os saudáveis.

A presença da doença na população de presas terá como resposta a diminuição de indivíduos suscetíveis, que por sua vez passam à classe de infectados. Aqui é considerada a forma de transmissão direta de parasitos, de um hospedeiro para o outro, ou seja, não houve necessidade de um vetor para a sua transmissão. Para a maioria das infecções, a taxa de contato aumenta proporcionalmente à densidade da população. Daí, a taxa de produção de novas infecções é igual a $\beta S I$, onde $\beta$ é o coeficiente de transmissão. Isso é chamado de transmissão dependente 
da densidade e também conhecido por lei da ação de massas. Considera-se que a doença leva à morte e de forma mais rápida que a morte natural, por isso desconsideramos a mortalidade das presas suscetíveis. Não ocorre recuperação uma vez que infectado. Escrevemos a mortalidade dos indivíduos infectados ocorrendo à taxa $d$.

Considerando a predação seletiva, é proposto que os predadores têm preferência pelos indivíduos suscetíveis, pois por alguma razão conseguem detectar quais indivíduos possuem ou não a doença. A diminuição que ocorre na variação populacional de presas suscetíveis é dada pela equação de Holling da resposta funcional do tipo 2 [5]. Essa resposta leva em consideração um tempo de manipulação de cada presa que o predador consome e ocorrerá a uma taxa de eficiência da predação $\omega$ e uma constante de saturação média $m$.

Em [3], se supõe que a variação da população de predadores ocorre logisticamente e aumenta com a resposta funcional da predação de indivíduos suscetíveis vezes a constante de eficiência da predação. Por essa razão resolvemos propor uma outra situação de variação da densidade populacional dos predadores ao problema. Consideramos que o crescimento da população de predadores depende da predação, onde $\epsilon$ é a constante de eficiência da predação, ou seja, a conversão de biomassa; vezes a equação da resposta funcional do tipo 2 como falamos anteriormente. O tempo de vida médio dos indivíduos da espécie não é tão grande quanto o tempo de vida médio da população de presas, logo consideramos a morte de indivíduos na população de predadores à uma taxa $e$.

Com base no que assumimos, obtemos o seguinte modelo matemático:

$$
\begin{aligned}
\frac{d S}{d t} & =r S\left(1-\frac{S+I}{K}\right)-\beta S I-\frac{\omega P S}{1+m S}, \\
\frac{d I}{d t} & =\beta S I-d I \\
\frac{d P}{d t} & =\epsilon \frac{\omega P S}{1+m S}-e P .
\end{aligned}
$$

O sistema será analisado com as seguintes condições iniciais: $S(0)>0 ; I(0)>0 ; P(0)>0$.

Escolhemos a seguinte mudança das variáveis:

$$
u=\frac{S}{K}, v=\frac{I}{K}, x=\frac{P}{K} \text { e } \tau=\beta t K
$$

e fizemos as seguintes mudanças de parâmetros:

$$
a=\frac{r}{\beta K}, b=\frac{\omega}{\beta}, c=m K, f=\frac{d}{\beta K} \text { e } g=\frac{e}{\beta K} .
$$

Substituíndo no sistema (1), as mudanças de variáveis e parâmetros conduzem a adimensionalização do sistema (1):

$$
\begin{aligned}
& \frac{d u}{d \tau}=a u(1-u-v)-u v-\frac{b u x}{1+u c}, \\
& \frac{d v}{d \tau}=u v-f v \\
& \frac{d x}{d \tau}=\epsilon \frac{b u x}{1+u c}-g x,
\end{aligned}
$$

em que $u(0)>0, v(0)>0, x(0)>0$. 


\section{Estudo da estabilidade}

Do ponto de vista biológico, nosso maior interesse é saber o que ocorrerá no sistema a longo prazo [7]. Por isso, queremos obter informações sobre o comportamento das soluções quando o tempo tende ao infinito. Para isso, faremos o estudo das soluções do sistema pelo primeiro método de Lyapunov [2]. Encontraremos os pontos de equilíbrio para saber o comportamento das soluções próximo desses pontos. É nesses pontos em que pode ocorrer a estabilidade das densidades das populações, quando o tempo $t$ cresce.

Para calcular os pontos críticos do sistema (2), assumimos que $\frac{d u}{d \tau}=0, \frac{d v}{d \tau}=0$ e $\frac{d x}{d \tau}=0$.

Resolvendo o sistema, encontramos quatro pontos de equilíbrio, $E_{0}=(0,0,0), E_{1}=(1,0,0)$, $E_{2}=\left(f, \frac{a(1-f)}{(a+1)}, 0\right), E_{3}=\left(\frac{g}{\epsilon b-c g}, 0, \frac{\epsilon a(\epsilon b-c g-g)}{(\epsilon b-c g)^{2}}\right)$.

Chamamos o ponto $E_{0}$ de solução trivial do sistema. Em termos biológicos, é quando ocorre a ausência de todas as populações. Em $E_{1}$, nota-se a presença apenas da população de presas suscetíveis, tanto a população de predadores quanto a de presas infectadas estão ausentes. Agora em $E_{2}$, se observa a presença apenas de presas, tanto de indivíduos suscetíveis como de infectados. E em $E_{3}$ temos a ausência de indivíduos doentes, uma situação de existência de predadores e presas suscetíveis. Portanto, apenas observando os pontos críticos, concluímos que não ocorre situação de coexistência das espécies com a presença da infecção, isso mostra a possibilidade de ocorrer uma aparente competição entre indivíduos infectados e predadores. A forma pela qual os parâmetros irão influenciar na configuração de cada um dos possíveis casos para as condições de estabilidade dos pontos críticos, segue enunciada nos seguintes teoremas:

Teorema 2.1 O ponto de equilíbrio $E_{0}$ é instável para toda combinação de parâmetros.

Teorema 2.2 $O$ ponto de equilíbrio $E_{1}$ é localmente assintoticamente estável se as seguintes condições forem observadas:

- $(1-f)<0$

- $\left(\frac{\epsilon b}{1+c}-g\right)<0$

Teorema 2.3 O ponto de equilíbrio $E_{1}$ é instável e $E_{2}$ localmente assintoticamente estável se as seguintes condições forem observadas:

- $(1-f)>0$

- $\left(\frac{\epsilon b}{1+c}-g\right)<0$

Teorema 2.4 $O$ ponto de equilíbrio $E_{1}$ é instável e $E_{3}$ localmente assintoticamente estável se as seguintes condições forem observadas:

- $(1-f)<0$

- $\left(\frac{\epsilon b}{1+c}-g\right)>0$

Teorema 2.5 O ponto de equilíbrio $E_{2}$ é instável e $E_{3}$ localmente assintoticamente estável se as seguintes condições forem observadas:

- $(1-f)>0$

- $\left(\frac{\epsilon b}{1+c}-g\right)>0$ 
- $\left(\frac{\epsilon b f}{1+c f}-g\right)>0$

Teorema 2.6 $O$ ponto de equilíbrio $E_{3}$ é instável e $E_{2}$ localmente assintoticamente estável se as seguintes condições forem observadas:

- $(1-f)>0$

- $\left(\frac{\epsilon b}{1+c}-g\right)>0$

- $\left(\frac{\epsilon b f}{1+c f}-g\right)<0$

Nas figuras (1) e (2), temos ilustradas as situações dos teoremas (2.5) e (2.6) respectivamante.

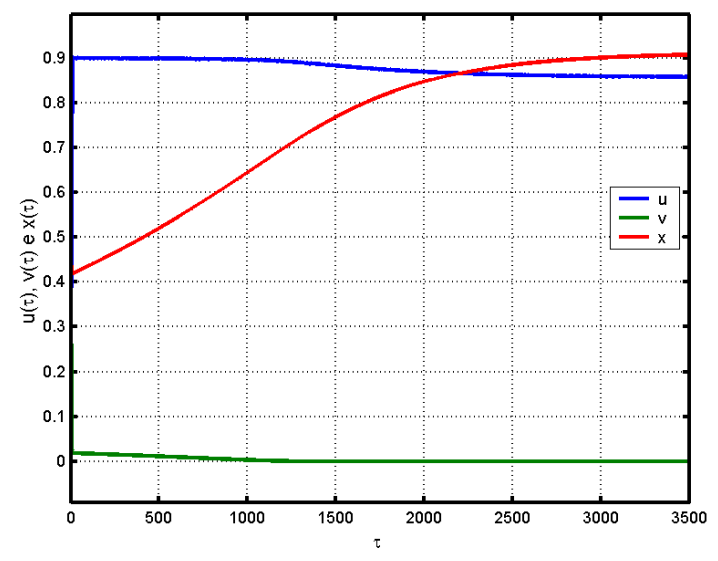

Figura 1: Estabilidade de $E_{3}$, temos que $(\epsilon b f / 1+c f)-g>0$. Foram utilizados os parâmetros: $r=0,1 ; K=50 ; \beta=0,002 ; \omega=0,003 ; m=0,2 ; \epsilon=0,67 ; d=0,09$ e $e=0,009$, sendo $u(0)=0,2 ; v(0)=0,499$ e $x(0)=0,456$ as condições iniciais.

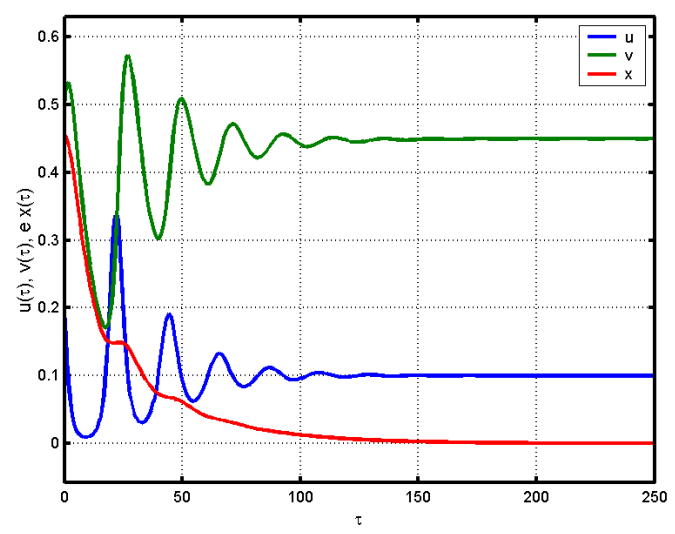

Figura 2: Estabilidade de $E_{2}$, temos que $(\epsilon b f / 1+c f)-g<0$. Foram utilizados os parâmetros: $r=0,1 ; K=50 ; \beta=0,002 ; \omega=0,003 ; m=0,2 ; \epsilon=0,799 ; d=0,01$ e $e=0,009$, sendo $u(0)=0,2 ; v(0)=0,499$ e $x(0)=0,456$ as condições iniciais.

Das duas primeiras relações que configuram os casos (2.5) e (2.6), escrevendo na forma 
correspondente dimensional, obtemos:

$$
\begin{aligned}
& K>\frac{d}{\beta}, \\
& K>\frac{e}{\epsilon \omega-e m},
\end{aligned}
$$

onde se observa a relação entre a capacidade de suporte da população de presas, $K$ e as taxas referentes aos indivíduos infectados e à população de predadores, $\frac{d}{\beta} \mathrm{e} \frac{e}{\epsilon \omega-e m}$ respectivamente. Ou seja, das configurações dos três grupos de valores formados por $K, \frac{d}{\beta} \mathrm{e} \frac{e}{\epsilon \omega-e m}$, obtivemos todas as situações referentes à estabilidade em cada um dos quatro ponto críticos.

Da relação $(\epsilon b f / 1+c f)-g>0$, escrita na forma correspondente dimensional:

$$
\frac{d}{\beta}>\frac{e}{\epsilon \omega-e m},
$$

se configura a região de estabilidade do teorema (2.5). Respectivamente para o teorema (2.6), invertendo a desigualdade. Logo, para a relação $(\epsilon b f / 1+c f)-g=0$, temos:

$$
\frac{d}{\beta}=\frac{e}{\epsilon \omega-e m},
$$

a igualdade de parâmetros que descreve a separatriz da região de equilíbrio. As relações obtidas das combinações dos parâmetros e a bifurcação ainda estão em estudo e serão abordadas em trabalhos futuros.

\section{Conclusões}

O modelo de Lotka-Volterra da competição interespecífica traz esclarecimento sobre os fatores determinantes da interação competitiva, $[4,8]$. Das principais conclusões do modelo, temos a possibilidade de interações fracas, onde podem as espécies coexistir, e fortes, onde uma das espécies acaba extinta. O princípio da exclusão competitiva [6] é observado pelo modelo quando a interação entre as espécies é agressiva, ou seja, forte.

Não havendo situação de pontos de equilíbrio na presença das três populações consideradas no sistema (2), já fomos levados à conclusão de não coexistência das três. Quando analisamos a estabilidade dos pontos críticos, obtivemos todas as possibilidades ecológicas para a situação modelada. Nas condições de (2.5) e (2.6) podemos afirmar a ocorrência de separatriz do estado de equilíbrio de $E_{2}$ para $E_{3}$ quando $(\epsilon b f / 1+c f)-g=0$ e esta situação ainda está em estudo. Com essas considerações, uma possível interpretação é a de ocorrência de competição entre a população de predadores e o parasito que gera a doença na população de presas.

A influência de um parasito na dinâmica populacional do hospedeiro ainda é uma questão não resolvida em ecologia de populações, pois mesmo sabendo da influência que os parasitos têm sobre taxas demográficas e que modelos matemáticos apontam o potencial de um parasito sobre a dinâmica do hospedeiro, ainda são incomuns dados reais sobre esse tipo de interação afetar de forma significativa, mas exemplos existem [1].

A aparente situação de competição é revelada pelo comportamento das soluções. Pensamos na população de presas suscetíveis na situação de recurso explorado pela população de predadores e pelo parasito responsável pela doença infecciosa, o que se configura como competição de exploração, pela interação indireta mediada pelo recurso compartilhado.

Sobre a predação com seleção e doença na presença de uma alimentação alternativa, a sobrevivência de um predador é dependente da dinâmica de uma comunidade de presas suscetíveis 
e infectadas. Como sempre, esse modo de predação afeta a dinâmica da população de presas infectadas por contato, de forma indireta. Nesse trabalho, observamos a não coexistência da população de predadores e da doença, ou seja, existem combinações de parâmetros nas quais a população de presas infectadas desaparece do meio. Esse é um dos casos, dentre todos os mostrados na nossa análise de estabilidade e exemplos numéricos, de fenomenos interessantes.

Nesse trabalho mencionamos que, sempre temos presente um número de situações reais de predação seletiva na presença de doenças, onde estudamos as restrições dos vários cenários pela análise teórica. Nosso estudo teórico pode mostrar a maioria de associações de mecanismos com aspectos epidemio-ecológicos onde a seleção de um predador evita a presa infectada dentro das considerações feitas. Experimentos futuros ou conjuntos de investigações nessa direção podem ajudar para verificar quando ou não, os processos prescritos por esse modelo funcionam atualmente num modo similar ao do mundo real.

\section{Referências}

[1] Begon, Michael; Townsend, Colin R.; Harper, John L., "Ecologia: de indivíduos a ecossistemas" (Tradução de Adriano Sanches Melo- 4. ed.), Artmed, Porto Alegre, 2007.

[2] Bobko, Nara, "Estabilidade de Lyapunov e Propriedades Globais para Modelos de Dinâmica Viral", Dissertação de Mestrado, PPGMA-UFPR, 2010.

[3] Das, Krishna pada; Roy, Shovonlal; Chattopadhyay, J., Effect of disease-selective predation on prey infected by contact and external sources, BioSystems, 95, (2009) 188-199.

[4] Edelstein-Keshet, Leah, "Mathematical Models in Biology", Society for Industrial and Applied Mathematics Philadelphia, USA, 2005.

[5] Holling, C. S., Some characteristics of simple types of predation and parasitsm, Canadian Entomologist, 91, (1959) 385-398.

[6] Holt, R. D.; Predation, apparent competition and the structure of prey community, Theoretical Population Biology, 12, (1977) 197-229.

[7] Ludwig, D.; Jones, D.D.; Holling, C.S., Qualitative Analysis of Insect Outbreak Systems: The Budworm and Forest, Journal of Animal Ecology, 47, (1978) 315-332.

[8] Murray, J. D., "Mathematical Biology", Third Edition, Springer, 2003. 